\title{
Ultra-Weak Photon Emission: A Promising Approach to Re- Interpret the Traditional Chinese Medicine
}

\author{
Xiaolei Zhao ${ }^{1}$ and Jinxiang $\operatorname{Han}^{1,2^{*}}$ \\ ${ }^{1}$ Department of Biochemistry and Molecular Biology, Shandong University, China \\ ${ }^{2}$ Shandong Medicinal Biotechnology Center, Shandong Academy of Medical Sciences, China
}

Submission: March 27, 2017; Published: May 05, 2017

*Corresponding author: Jinxiang Han, Shandong Medicinal Biotechnology Center, Key Laboratory for Biotech-Drugs of the Ministry of Health, Shandong Academy of Medical Sciences, Jinan 250062, Shandong Province, China, Tel: +86-053182919608; Email: samsjxhyx@163.com

\begin{abstract}
Traditional Chinese medicine (TCM) is an ancient medical practice system. The theory of it is deeply rooted in the Chinese culture and has a heavy philosophical bent. The abstract theories, fuzzy concepts, and subjective examination methods make the development of the TCM remain stagnant. Therefore, how to free the theory of TCM from heavy philosophy to achieve separation of medicine and philosophy is a way for TCM to get out of the woods. In this article, we introduced the Ultra-weak photon emission (UPE) from the human body and discussed the relationship between the coherent theory of UPE and TCM theory. On this basis, together with some research results of UPE, we proposed the Meridian hypothesis, Viscera-state hypothesis, hypothesis of correspondence between man and nature, and Syndrome differentiation hypothesis. Finally, we conferred the theory of TCM a new image - the Quantum TCM theory. Although some hypothesises need further experimental verification, the UPE and its coherent theory provide a promising approach to re-interpret the TCM.
\end{abstract}

Keywords: Traditional chinese medicine; Ultra-weak photon emission; Coherent theory of UPE

Abbreviations: TCM: Traditional chinese medicine; UPE: Ultra-Weak Photon Emission

\section{Introduction}

Traditional Chinese medicine (TCM), as an ancient medical practice system, has a history of more than two thousand years, and it has made tremendous contributions to the medical service in China. The holism thought and syndrome differentiation are the two major features and advantages of the TCM. In understanding diseases, TCM emphasizes on integrity and individual parts of the body, the general and specific character, and the body's interrelationship with its natural environment. Therefore, satisfactory treatment results can be achieved generally. However, the theoretical system of TCM is deeply rooted in the Chinese culture and has a heavy philosophical bent. The abstract theories, fuzzy concepts, and subjective examination methods make the development of the TCM remain stagnant [1].

Although substantial efforts have been made in recent years to research and practice in the modernization of TCM, and have achieved some results [2,3], a qualitative breakthrough has not yet achieved due to the reason that these researches ignore the thinking mode of ontology with the integrity and organic characteristics in TCM. Given this background, it is of great significance to develop a new approach which can re- interpret the traditional Chinese medicine using contemporary scientific language and realize the conversion of TCM theory into a scientific paradigm based on TCM's own laws and modes of thinking to make the full use of the advantages of TCM in preventing and treating diseases. Based on it, we introduced the Ultra-weak photon emission (UPE) and its coherent theory, and discussed its feasibility to re-interpret the TCM in this paper.

The Commensurability of Philosophic Thought of TCM and UPE

The UPE, as an intrinsic attribute of living system, originates from the electron transition of biological molecules from a high energy state to a low energy state during oxidative metabolic processes. It belongs to the category of quantum. It is known that, the complementary principle is the key thought of quantum theory. And its essence is the unity of opposites, which is greatly influenced by the Taiji graph of Taoism and is consistent with the principle of yin-yang balance in TCM theory. Thus, the UPE shares the similar philosophic thought with the TCM.

The coherent theory of UPE put forward by Popp holds that there are non-locality coherent electromagnetic fields in biological systems [4]. And the coherent electromagnetic 
radiations (namely UPE) from the electromagnetic fields are the basis for communication between different cells, tissues and organs in biological systems [5] and can reflect the order of life as a whole which is consistent with the whole concept of TCM. Any changes in biological system, whatever it is a component or a structure, will cause changes at the microscopic energy level, leading to changes of UPE. It is naturally expected that these properties of UPE are similar with that of "Qi" in TCM. Therefore, it is possible to translate the TCM theory using the coherent theory of UPE.

Based on this and combining the common physical features of coherent electromagnetic radiations (UPE) and "Qi", we proposed a view that the coherent electromagnetic radiation from the body could characterize the human "Qi" in TCM, and gave the theory of TCM a new image-the Quantum TCM theory [6].

\section{The Connotation of the Quantum TCM}

\section{Meridian hypothesis}

A reduced theoretical model for the coherence of UPE in human body was used in our lab to search for the relationship between the coherent electromagnetic radiations (UPE) and the meridian [7]. Our results indicated that the electromagnetic radiations from cells (biomacromolecule, tissues, or organs, etc.) in different location of body could form interference in the body and crisscross electromagnetic bunching in the surface of body. And we found that the location of electromagnetic bunching basically coincided with that of meridian. The studies from Yan et al. [8] also found that fourteen luminescence beams in the surface of body were coincident with fourteen meridians. Based on it, we proposed the Meridian hypothesis: meridian was the integral stereo network fringe (bunching) formed by the coherent electromagnetic fields in body.

\section{Viscera-state hypothesis}

Many researches confirmed that the UPE could be a messenger to deliver the biological information between different cells, tissues, and organs, and was the basis for communication in living systems [5]. This function of UPE is consistent with that of "Qi" in Viscera-state doctrine of TCM which thinks that "Qi" as a messenger contacts every viscera, orifices, and even the nature through the meridian. Thus, we proposed the Viscera-state hypothesis: coherent electromagnetic radiation of organism was another messenger to contact with various tissues, organs, cells, and biomacromolecules, and was similar with the "Qi" to regular the viscera network [9].

\section{The hypothesis of correspondence between man and nature}

The lives on earth swim in a spatio-temporal electromagnetic field, and the coherent electromagnetic fields in bodies are produced and sustained under the background of spatio-temporal electromagnetic field. Therefore, there must be a resonance between both magnetic fields, which maintains a balance. When the spatio-temporal electromagnetic field changes, the balance will be broken. And they will bring some impacts on the human body. Interestingly, this phenomenon is no stranger to us, such as the biological rhythms. Thus, we think that the resonance between both magnetic fields could be considered as the "Qi" of the universe, and the "correspondence between man and nature" is a resonant interaction between the coherent electromagnetic field of human bodies and the spatio-temporal electromagnetic field [10].

\section{Syndrome differentiation hypothesis}

As we discussed above, the coherent electromagnetic radiations (namely UPE) from the body could characterize the human "Qi" in TCM and reflect the order of life as a whole. And any small changes in body will lead to the changes of UPE [11]. Therefore, we speculated that the quantum state of the UPE of the body should be able to represent the state of the organism, namely the syndrome. Actually, many studies have confirmed this view. For example, based on the yin-yang balance theory in TCM, Yang et al. found that the intensities of UPE from the left and right hands were balanced in normal people, while they were imbalanced in stroke patients [12]. Some interesting phenomena have been found in our lab: the intensity of UPE from the first finger (lung channel passing this finger) of the patient with Qi deficiency were obviously lower than that of healthy patients; and the similar phenomena were also found in the middle finger (pericadium meridian passing this finger) and little finger (heart channel of Hand-Shaoyin passing this finger) of the patient with insufficiency of the heart-Qi. Based on these facts, we proposed the Syndrome hypothesis: the symptom in TCM was the quantum superposition state formed by the coherent electromagnetic radiation of body, and syndrome differentiation was the treatment principle which cured the patients through adjusting the quantum superposition states of coherent electromagnetic fields.

\section{The concept of Quantum TCM}

Based on the above ideas and the view that the coherent electromagnetic radiation from the body could characterize the human "Qi" in TCM, the concept of Quantum TCM is put forward: The Quantum TCM is a subject which research and interpret the TCM theory considering the transformation rules of human health and diseases and their prevention, diagnosis, therapy, rehabilitation and healthcare using the quantum forms of electromagnetic radiation, photon (quantum) radiation, energy (caloric), etc. Additionally, it uses the holism and dialectical materialism as its philosophical thought, and the quantum theory (especially, the coherent theory of UPE) as its theoretical basis.

The Quantum TCM, based on the coherent theory of UPE, frees the TCM theory from heavy philosophy and re-interprets it using the contemporary science language based on TCM's own laws and modes of thinking, which makes the abstract concepts in TCM theory obvious, the fuzzy law clear, and subjective 
examination methods objective. Although some hypothesises need further experimental verification, the UPE and its coherent theory provide a promising approach to re-interpret the traditional Chinese medicine.

\section{References}

1. Zhao XL, Han JX (2013) The connotation of the Quantum Traditional Chinese Medicine and the exploration of its experimental technology system for diagnosis. Drug Discov Ther 7(6): 225-232.

2. Huang X, Chen Q Yang G, Dai W, Lang Q et al. (2012) Metabolic Profiling Study of Yang Deficiency Syndrome in Hepatocellular Carcinoma by H1 NMR and Pattern Recognition. Evid Based Complement Alternat Med 2012: 843048.

3. Zhang NL, Yuan S, Chen T, Wang Y (2008) Statistical validation of traditional chinese medicine theories. J Altern Complement Med 14(5): 583-587.

4. Popp FA (2003) Properties of biophotons and their theoretical implications. Indian J Exp Biol 41(5): 391-402.

5. Vanwijk R (2001) Bio-photons and Bio-communication1. Journal of Scientific Exploration 15(2): 183-197.

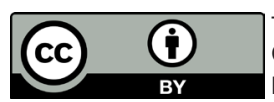

This work is licensed under Creative Commons Attribution 4.0 Licens

DOI: 10.19080/JCMAH.2017.02.555584
6. Han JX (2011) Try to discuss the modernization of TCM basic theory. Medical Innovation of China 8(2): 184-189.

7. Han J (2012) Meridian is a three-dimensional network from bioelectromagnetic radiation interference: an interference hypothesis of meridian. Cell Biochem Biophys 62(2): 297-303.

8. Yan ZQ Wang YZ (1989) A study on the characteristics of luminescence from fourteen meridians of human body. Acupuncture research (3): 389-394.

9. Han JX, Han Y (2011) A viscera-state doctrine based on the physical and chemical perspective. Journal of Shandong University of TCM 35(1): 17-19.

10. Han JX, Han Y (2011) Try to discuss the scientific connotation of "heaven-human correspondence". Journal of Shandong University of TCM 35(2): 63-65.

11. Zhao X, Pang J, Fu J, Wang Y, Yang M, et al. (2017) Spontaneous photon emission: A promising non-invasive diagnostic tool for breast cancer. J Photochem Photobiol B 166: 232-238.

12. Yang JM, Choi C, Hyun-hee, Woo WM, Yi SH, et al. (2004) Left-right and Yin-Yang balance of biophoton emission from hands. Acupunct Electrother Res 29(3-4): 197-211.

\section{Your next submission with Juniper Publishers will reach you the below assets}

- Quality Editorial service

- Swift Peer Review

- Reprints availability

- E-prints Service

- Manuscript Podcast for convenient understanding

- Global attainment for your research

- Manuscript accessibility in different formats

( Pdf, E-pub, Full Text, Audio)

- Unceasing customer service

Track the below URL for one-step submission https://juniperpublishers.com/online-submission.php 